\title{
Açık Kaynaklı Gezgin Robotların Mühendislik Eğitiminde Kullanımı ve Örnek Bir Müfredat
}

\author{
Ömer Mutlu Türk KAYA ${ }^{\text {a1* }}$ \\ a İstanbul Sabahattin Zaim Üniversitesi, Mühendislik ve Doğa Bilimleri Fakültesi, Yazılım Mühendisliği Bölümü, İstanbul, Türkiye \\ İstanbul Sabahattin Zaim Üniversitesi Fen Bilimleri Enstitüsü Dergisi (2021) 3 (1): 01-10 \\ https://doi.org/10.47769/izufbed.866093 \\ (iD) $O R C I D{ }_{1} 0000-0003-0859-3261$
}

\begin{tabular}{l}
\hline YAYIN BİLGİṠ \\
\hline Yayın geçmişi: \\
Gönderilen tarih: 21 Ocak 2021 \\
Kabul tarihi: 27 Ocak 2021 \\
\end{tabular}

Anahtar kelimeler:

Açık Kaynak

Gezgin Robot

Mühendislik Eğitiminde Gezgin

Robotlar

Örnek Gezgin Robot Müfredat

\section{ÖZET}

Dünya üzerindeki robotlaşmanın artmasıyla ve bilginin özgürce yayılmasıyla birlikte robot bilimi alanı genişlemekte, mevcut alt alanlarının sayısı ve kaynakları her geçen gün artmaktadır. Eş zamanlı olarak da kaynakların açık, özgür bir şekilde kullanılma bilinci gelişmektedir. Bu çalışmada eğitim amacıyla geliştirilen açık kaynaklı gezgin robotlar ve onların mühendislik eğitiminde kullanımı incelenmiştir. Bunun yanında mühendislik eğitimi veren üniversitelerin gezgin robotlarla ilgili müfredatları ve ders işleme yöntemleri incelenerek bir dönemlik, örnek gezgin robotlar ders planı ve içeriği hazırlanmıştır. Bu kapsamda uluslararası ve ulusal düzeyde pek çok üniversite incelenmiştir.

\section{Usage of Open-Source Mobile Robots in Engineering Education and a Sample Curriculum}

\begin{tabular}{l}
\hline ARTICLE INFO \\
\hline Article history: \\
Received: 21 January 2021 \\
Accepted: 27 January 2021 \\
\hline Key words: \\
Open Source \\
Mobile Robot \\
Mobile Robots in Engineering \\
Education \\
Sample Mobile Robot \\
Curriculum \\
\end{tabular}

\section{Giriș}

Bilim ve teknoloji dünyasındaki gelişmelere paralel olarak robot bilimindeki gelişmelerin de her geçen gün artmasıyla ve özellikle açık kaynak bilincinin insanlarda yerleşmesiyle birlikte uzak gelecek gibi görünen robotların mühendislik

\begin{abstract}
With the increase of robotization in the world and the free spread of information, the field of robotics is expanding, the number and resources of its existing subfields are increasing day by day. Simultaneously, the awareness of using resources in an open and freeway is developing. In this study, open-source mobile robots developed for educational purposes and their use in engineering education were examined. In addition, by examining the curricula and teaching methods of mobile robots of universities that provide engineering education, a one-term, sample mobile robots syllabus and course content were prepared. In this context, many universities at international and national level have been examined.
\end{abstract}

eğitiminde kullanılması günümüzde önemli ölçüde yaygınlaşmıştır. Disiplinler arası bir bölüm olan robot biliminin yanı sıra temel mühendislik disiplinlerinde de eğitim veren öğretim görevlileri, araştırmacılar ya da daha kapsamlı ifade etmek gerekirse bilim adamları ürettikleri ve öğretecekleri teorik bilgileri deneyebilmek ve öğrencilerine de 
denetebilmek için gerçek robotlara ihtiyaç duymaya başlamışlardır. Ancak deneylerin yapılacağı gerçek robotların özgürce incelenmesi, üretilmesi, istenildiği takdirde belirli bölümlerinin değiştirilmesi, güncellenmesi, yeni özelliklerin eklenmesi veya gereksiz olanların çıkarılması, paylaşımının ve dağıtımının yapılabilmesi gibi işlemlere ve müdahalelere açık olması gerekmektedir. Buna bilimsel ve teknolojik yazınlarda açık kaynak denmektedir. Kısaca açık kaynak kavramından ve tarihsel sürecinden bahsetmek gerekirse; İngilizcedeki "open source" teriminin Türkçe karşılığıdır. Ancak açık kaynak bilinci "open source" kavramının ortaya çıkmasıyla değil, Richard Stallman'ın 1983 yılında GNU (GNU's Not Unix) Projesi aracılı̆̆ıyla "free software" yani özgür yazılım kavramını ortaya çıkarmasıyla başlamıştır. Ancak "free" sözcüğü, İngilizce'de hem "özgür" hem de "bedava" anlamına geldiğinden bu kavramla ilk defa karşılaşan insanlarda kafa karışıklığı yaratıyordu. Kafa karışıklığını gidermek amacıyla bu kavramı tanıtırken şu meşhur cümleyi kullanıyorlard1: "We mean free as in freedom, not free as in beer ("How I coined the term 'open source'। Opensource.com", y.y.). Yani özünde denilmek istenen "free" sözcüğünün "bedava bira" söz öbeğindeki "bedava" olarak değil, "özgürlük" sözcüğündeki "özgür” olarak kullanıldığıdır. Ancak gelinen bu noktayı Christine Peterson şu şekilde açıklamaktadır: "Bu noktada, yazılım üzerindeki tartışma alkollü bir içeceğin fiyatına dönüştü. Sorun, "free software" kavramının açıklanmasının olanaksız olması değildi. Sorun, böyle önemli bir düşünce için seçilen bir adın yeni katılanlar açısından çok fazla kafa karıştırıcı olmaması gerekliliğiydi. Daha net ve anlaşılabilir bir ada, terime ihtiyaç vard1." ("How I coined the term 'open source' | Opensource.com”, y.y.). Böylelikle 1998 yılında, Christine Peterson, arkadaşları Eric Raymond, Brian Behlendorf, Michael Tiemann, Todd Anderson, Mark S. Miller ve Ka-Ping Yee ile yaptığı toplantıların birinde "open source" yani "açık kaynak" kavramını ortaya attı. Diğerleri tarafından benimsenen bu ad, sonraki hafta yapılan beyin firtınası toplantısında tanıtıld1. Akabinde www.opensource.org sitesi kuruldu ve böylelikle "açık kaynak" kavramı yayılmaya, insanlar tarafindan kullanılmaya başlandı. Bütün bu gelişmelerin 1şı̆̆ında açık kaynak hareketinin fikir babası, "free software" kavramının yaratıcısı olan Richard Stallman'ın ilkesel olarak "open source (açık kaynak)" kavramı ile aynı fikirde olmadığını ve halen "free software (özgür yazılım)" kavramını savunduğunu bir dipçe olarak belirtmekte fayda var ("About the GNU Project - GNU Project - Free Software Foundation", y.y.). İngilizce "free" sözcüğünün yol açtığı bu kavram karmaşasını ve bu karmaşanın bir düşünceyi adeta 2 farklı kola bölmesini göz önüne aldığımızda kavramların her ikisini de güzel Türkçemiz ile ifade ettiğimiz zaman gayet anlaşılır olduğunu görmekteyiz. Bu çalışmada kavram karmaşasına neden olmamak adına özgür yazılım ve açık kaynak kavramlarının tek bir düşünceyi, bilinci, hareketi anlattığı kabul edilmiştir. $\mathrm{Bu}$ nedenle bu düşünce, bilinç ve hareketi ifade etmek için çalışmanın genelinde "açık kaynak" terimi kullanılmıştır. Açık kaynak kavramı bir yazılım programının kaynak kodlarıyla ilintili olarak ortaya çıksa da günümüzde daha geniş bir anlama, yola, yönteme ve bilince dönüşmüştür. İnsanların özgürce kullanımına sunulan bilgisayar kodlarının dışında, bir ürünün aynı nitelikte tekrar özgürce üretilebilmesi, paylaşılabilmesi ve dağıtılabilmesi için gereken 3 boyutlu tasarım dosyaları, kullanılan malzeme bilgisi, üretim ve montaj yöntemleri vb. gibi bölümleri de açık kaynak kapsamında değerlendirilmektedir. Bu çalışmada kapsamı daraltıp, niteliği artırmak adına genel olarak robotların mühendislik eğitiminde kullanımının incelenmesinden ziyade özel olarak açık kaynaklı gezgin robotların ve açık kaynaklı gezgin robotların mühendislik eğitiminde kullanımının incelenmesine yoğunlaşılmıştır. Dolayısıyla 2. bölümde açık kaynaklı gezgin robotlar ve açık kaynaklı gezgin robotların mühendislik eğitiminde kullanımı, 3. bölümdeyse incelenen ders müfredatlarının genel bir özeti ve ayrıntılarıyla birlikte örnek bir müfredat sunulmuştur. Çalışmada varılan sonuçlar 4. bölümde, kaynaklar ise son bölümde verilmiştir.

\section{Açık Kaynaklı Gezgin Robotların Mühendislik Ĕgitiminde Kullanımı}

Açık kaynak bilincinin yazılım alanının dışına taşmasıyla birlikte kapalı kaynak, salt ticari amaçlarla üretilen gezgin robotların dışında, Ar-Ge yapmak ve mühendislik eğitiminde kullanmak amacıyla açık kaynaklı gezgin robotlar üretilmeye başlanmıştır. Hatta, üretilen bu robotlar her geçen gün değişmekte, gelişmekte, sayıları artarak kullanımı yaygınlaşmaktadır. Günümüzde gezgin robotlar iç ve dış mekanlarda servis ve hizmet robotu olarak, savunma ve güvenlik alanında askeri unsur, bomba imha robotu olarak, esatış firmalarının, üretim ve depolama yapan firmaların depolarında ürün taşıyan araçlar olarak, havacılık sektöründe uçak gövdelerini taşıyan araçlar olarak ve benzeri birçok sektörde aktif bir şekilde kullanılmaktadır. Her geçen gün bu alanların sayısı artmakta ve ihtiyaç duyulan gezgin robot platformları değişmektedir. Üniversiteler, bu alandaki donanımlı mühendis ihtiyacını düşük maliyet ve yüksek faydayla karşılamak için açık kaynaklı gezgin robotlar üzerine yoğunlaşmakta ve müfredatlarını günbegün geliştirmektedirler. $\mathrm{Bu}$ çalışmada ulusal ve uluslararası, toplamda 59 üniversite incelenmiş ve mühendislik eğitiminde kullanılan gezgin robotlar tespit edilmiştir. Bunların en bilineni ve yaygını Turtlebot'tur. Bu robotlar Melonee Wise ve Tully Foote tarafindan Kasım 2010 yılında Willow Garaj1'nda oluşturulmuştur ("About - TurtleBot”, y.y.). Uygun bir maliyeti olan bu robotlar 2 adet DA motor tahrikli ve 1 adet sarhoş olmak üzere 3 adet tekerden oluşan bir gövdeden oluşmaktadır. Üzerinde çeşitli mesafe sensörleri, çarpışma sensörü ve kamera bulunan bu robotlara yeni sensörler kolayca eklenebilmektedir. Turtlebot' un 3 versiyonu vardır. Şekil 1 'de de görüldüğü gibi bunlar ; Turtlebot1, Turtlebot 2 ve Turtlebot 3 'tür.

Tamamen açık kaynak kodlu olan Turtlebot1 ve Turtlebot2‘ye ek olarak, Turtlebot3 açık kaynak kodlu olmasının yanında 3B tasarım modelleri de herkese açık bir şekilde internet sitesinden paylaşılmaktadır (“Open Source - TurtleBot”, y.y.). Turtlebot1 ilk üretilen Turtlebot'tur ve iRobot Create tabanına, $10800 \mathrm{C}$ batarya paketine sahiptir. Bunun yanında jiroskop ve Kinect destekli bir güç kartı ve 2 çekirdekli Asus marka bir laptopa sahiptir. Turtlebot2 ise Yujin Kobuki tabanı üzerine kurulmuş, $7920 \mathrm{C}$ batarya paketi, 1 adet Kinect sensör, 1 adet Asus laptop, hızlı şarj ve şarj istasyonu gibi özelliklere sahip olup, Ekim 2012 yılında piyasaya sürülmüştür. Turtlebot3 ise önceki versiyonlara göre daha küçük, fakat daha fazla özelliğe sahiptir. Mayıs 2017 yılında piyasaya sürülen bu robot, modüler yapısıyla şekli değişebilir bir yapıdadır. 3 farklı model olarak üretilmiştir. Bunlar: Burger, 
Waffle and Waffle $\mathrm{Pi}^{\text {‘d }}$ ir. 2 adet DA motora, $6480 \mathrm{C}$ batarya paketine, 1 adet 360 derece Lidara, 1 adet kameraya sahip olan Burger'in özelliklerine ek olarak Waffle 1 adet RealSense kamera barındırırken, Waffle Pi ise Raspberry Pi kamera barındırmaktadır. Bunların yanında Raspberry Pi ve Intel Joule 570xx tek kart bilgisayarlar barındırmaktadır ("About TurtleBot", у.у.). Turtlebotlar, uluslararası düzeyde Southern California Üniversitesi, Washington Üniversitesi (St Louis), Brown Üniversitesi, KU Leuven Üniversitesi, ulusal düzeyde de Kocaeli Üniversitesi, Sakarya Uygulamalı Bilimler Üniversitesi ve İstanbul Sabahattin Zaim Üniversitesi gibi pek çok üniversite tarafından mühendislik eğitiminde ve araştırma amaçlı kullanılmaktadır. Tabii ki Turtlebot'un dışında da açık kaynaklı çeşitli gezgin robotlar geliştirilip mühendislik eğitiminde kullanılmaktadır. Bunların en önemlilerinden biri Boulet, Guldner, Lin ve Karaman tarafindan 2014 y1lında geliştirilmeye başlanan MIT robot yarış arabasıdır ("racecar", у.у.).

\section{Original TurtleBot}

ionetentinumed

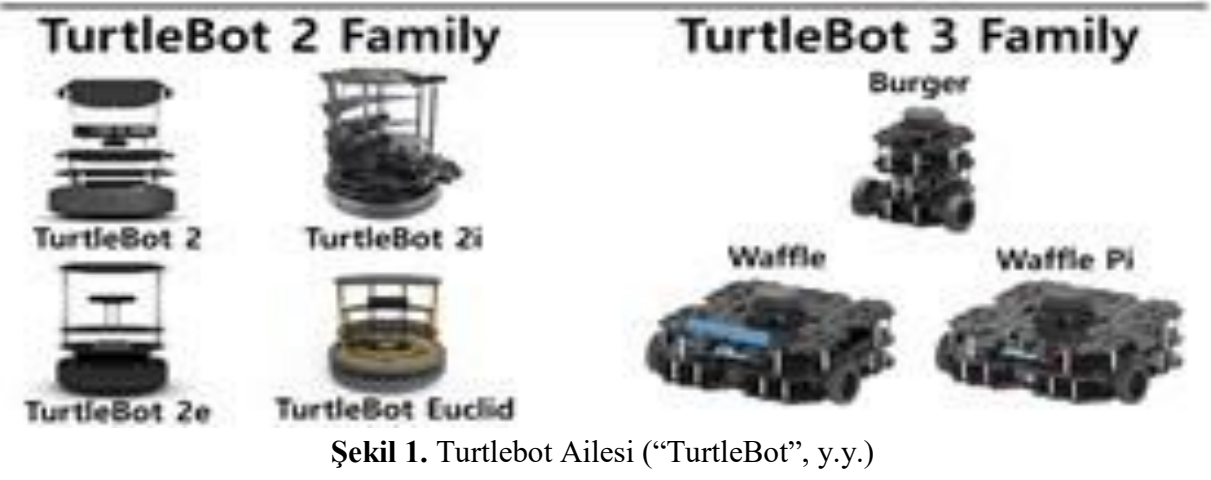

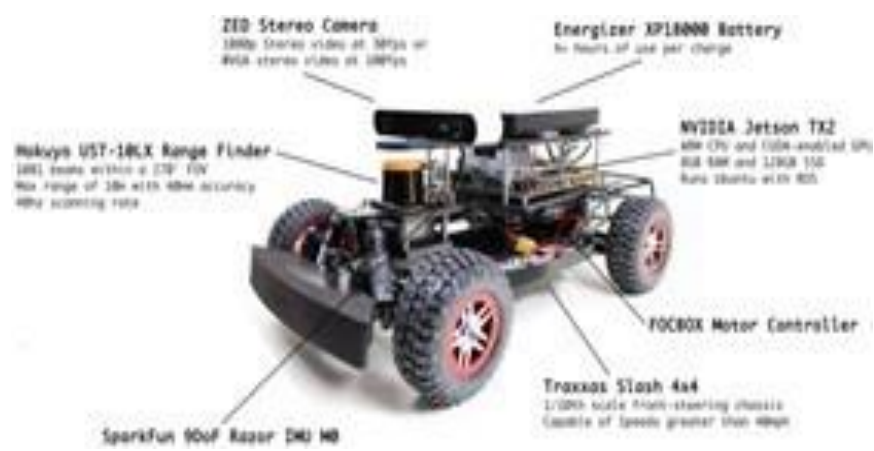

Şekil 2. MIT Robot Yarış Arabası ("Platform”, y.y.)

Geliştirilen bu robotlar, MIT 'de pek çok derste ve araştırmada defalarca kullanılmıştır ve halen de kullanılmaya devam etmektedir. Bu robotlar, 2016 y1lından beri verilen "Robot Bilimi: Bilimler ve Sistemler" adındaki MIT'nin lisanstaki amiral gemisi sayılan derse önemli ölçüde güç katmıştır ("racecar", у.у.). Tamamen açık kaynaklı olan robotun tüm dosyaları ("mit-racecar · GitHub", y.y.) 'deki GitHub deposunda bulunmaktadır. Srinivasa ve ark. MIT Robot Yarış Arabas1 Projesinden esinlenerek (Srinivasa vd., 2019)‘da Washington Üniversitesi, Paul G. Allen Bilgisayar Bilimi ve Mühendislik Okulundaki Kişisel Robot Bilimi Laboratuvarında düşük maliyetli, tamamıyla açık kaynaklı MuSHR (Multi-agent System for non-Holonomic Racing) robot yarış arabasını geliştirmişlerdir. Herkese açık belgeleriyle ve öğretici, kendin yap kılavuzlarıyla kolayca yapılabilen bir gezgin robottur. Washington Üniversitesi ‘nde, Gezgin Robotlar dersinde kullanılmak amacıyla çeşitli örnekler, laboratuvar ödevleri hazırlanmış olup, aktif olarak kullanılmaktadır. Gezgin robotlar alanında yoğunlaşan üniversitelerin laboratuvarları ve robot bilimciler için önemli bir araç olan MuSHR şekil 3 'de görülmektedir. Ayrıntılı bilgi için https://mushr.io adresi ziyaret edilebilir ve açık kaynak kodlarına da https://github.com/prl-mushr sitesinden erişilebilir.

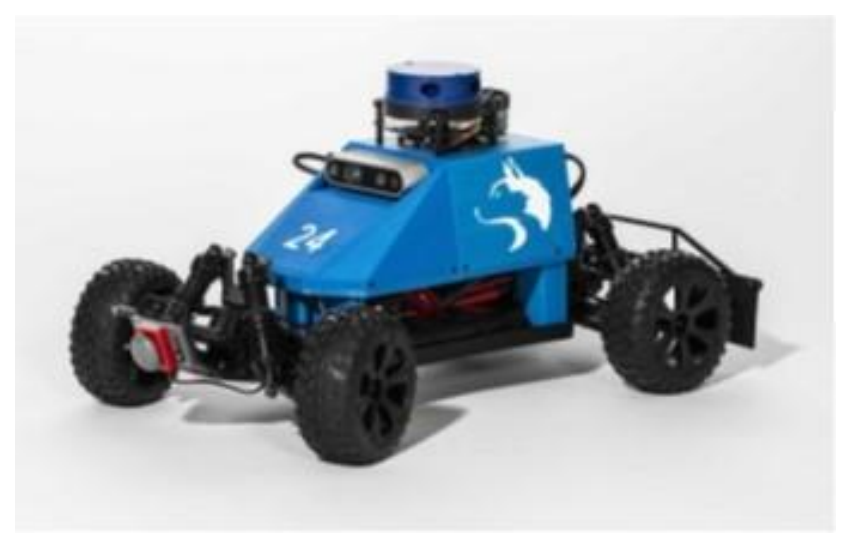

Şekil 3. MuSHR Robot Yarış Arabası (Srinivasa vd., 2019)

Canals ve arkadaşları (Albo-Canals, Garcia-Casulleras, De Cordoba, Canaleta, \& Gonzalez-Dachs, 2014) tarafindan La Salle - Ramon Llull Üniversitesinde tasarlanıp geliştirilen LSMaker EV1, bir önceki LSMaker versiyonuna göre Arduino tabanlı bir donanıma sahip, açık kaynaklı olup, tüm mühendislik müfredatlarında kolaylaştırıcı bir unsur olarak kullanılması hedeflenmiştir. Akçakoca, yüksek lisans tezinde (Mehmet Akçakoca, 2017) eğitim ve araştırma amaçlı Evarobot'u geliştirmiştir. Şekil 4 'te görülen Evarobot, ROS (Robot Operating System) ara katmanı kullanılarak geliştirilmiş olup, yazılım kütüphaneleri de açık kaynak olarak internet üzerinden paylaşılmıştır. 


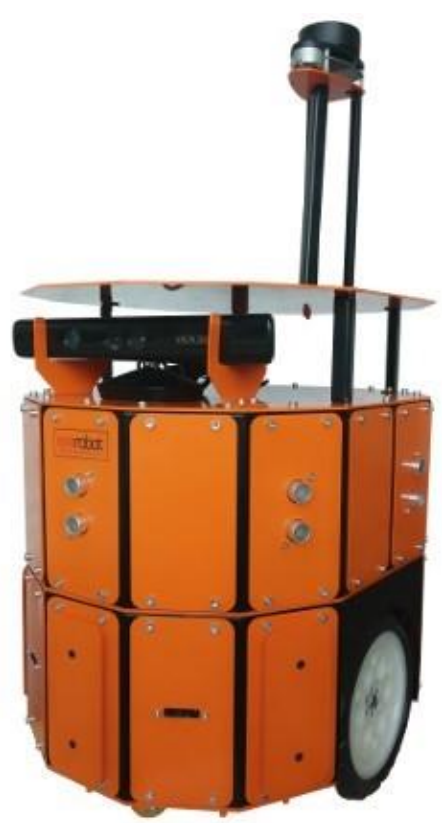

Şekil 4. Evarobot (“Evarobot”, y.y.)

Evarobot‘un üniversitelerin mühendislik eğitiminde kullanımıyla ilgili bir bilgiye ulaşılamamıştır. (Adinandra, Nofriyudi, Pratama, \& Ratnawati, 2018)`de Adinandra ve arkadaşları, Endonezya'da eğitim ve araştırma alanında kullanılmak üzere CEPI adında, diferansiyel ve omni sürüş tipinde iki açık kaynak kodlu gezgin robot geliştirmişlerdir. Ancak bu robotların herhangi bir üniversitede, mühendislik eğitiminde kullanımıyla ilgili bir bilgiye ulaşılamamıştır. Arvin ve arkadaşları (Arvin vd., 2019)'da düşük maliyetli, kullanımı kolay, yazılım, donanım anlamında tamamıyla açık kaynaklı olan Mona gezgin robotunu geliştirdiler. Mona, Manchester Üniversitesi‘nde hem mühendislik eğitiminde temel kontrol sistemleri, PID denetleyici vb. konuları öğretmek için hem de çeşitli bilimsel araştırmalarda başarılı bir şekilde kullanılmıştır.

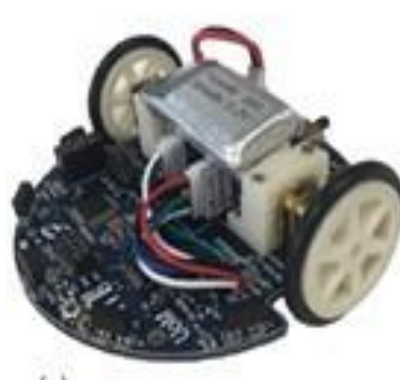

(a)

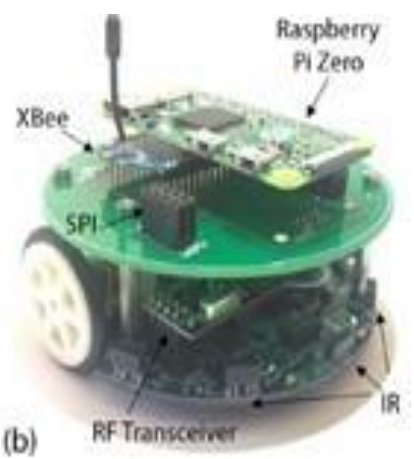

(b)
Şekil 5. a) Mona Robot, b) Geliştirme kartlarıla birlikte Mona Robot (Adinandra vd., 2018)

Manchester Üniversitesi, Elektrik- Elektronik Mühendisliği Okulu bu robotları 2 şekilde kullanmaktadır. İlki; dolaylı olarak bütün lisans öğrencileri için uygulamalı grup proje ödevi anlamında, ikincisiyse; Mekatronik Mühendisliği lisans ve yüksek lisans ders müfredatlarında doğrudan Gezgin Robotlar ve Özerk Sistemler dersi kapsamındadır. Öğrenciler bu şekilde gezgin robot kinematiği, algılama, planlama, yön bulma, tahminde bulunma vb. konularda deneyim kazanmaktadırlar. Arvin ve arkadaşları, Mona'nın yanı sıra (Arvin vd., 2019)'da, eğitim amaçlı kullanılan bazı gezgin robotların karşılaştırmasını yapmıştır. Tablo Şekil 6`da verilmiştir.

\begin{tabular}{llllll}
\hline Robot & Release date & Cost & Research or education & Open source & Commercialised \\
\hline Aeris & 2017 & Unknown & Education & Yes & No \\
Andruino-Al & 2016 & $£ 30$ & Education & Yes & No \\
Colias & 2014 & $£ 25$ & Research & Yes & No \\
E-puck & 2009 & $£ 700$ & Both & Yes & Yes \\
Jasmine & 2006 & Unknown & Research & Yes & No \\
Khepera IV & 2016 & $£ 3.5 k$ & Both & No & Yes \\
MarXbot & 2009 & Unknown & Research & Yes & No \\
microMVP & 2017 & $£ 70$ & Both & Yes & Yes \\
Rice r-one & 2013 & $£ 200$ & Both & Yes & No \\
Thymio II & 2010 & $\$ 105$ & Education & Yes & Yes \\
\hline
\end{tabular}

Şekil 6. Eğitim amaçlı kullanılan bazı gezgin robotların karşılaştırılması (Adinandra vd., 2018)

(Cianci vd., 2009)`da Mondada ve ark. özellikle mühendislik eğitiminde kullanılması amacıyla yazılım, donanım ve kılavuzlar bağlamında tamamen açık kaynaklı E-Puck robotunu sunmuşlardır. Halihazırda, CGtronic ve AAI Japan adlı iki şirket E-Puck gezgin robotunu üretmekte ve açık donanım lisansı altında 10 firma dağıtımını yapmaktadır (Cianci vd., 2009).

E-Puck gezgin robotları, EPFL (Ecole Polytechnique Federale De Lausanne)'de Sinyal İşleme, Otomatik Kontrol, Davranış Tabanlı Robot Bilimi, Dağıtık Zeki Sistemler, Bir Gezgin Robotun Konum Tahmini ve Yol Bulması gibi konuların öğretilmesinde kullanılmaktadır.

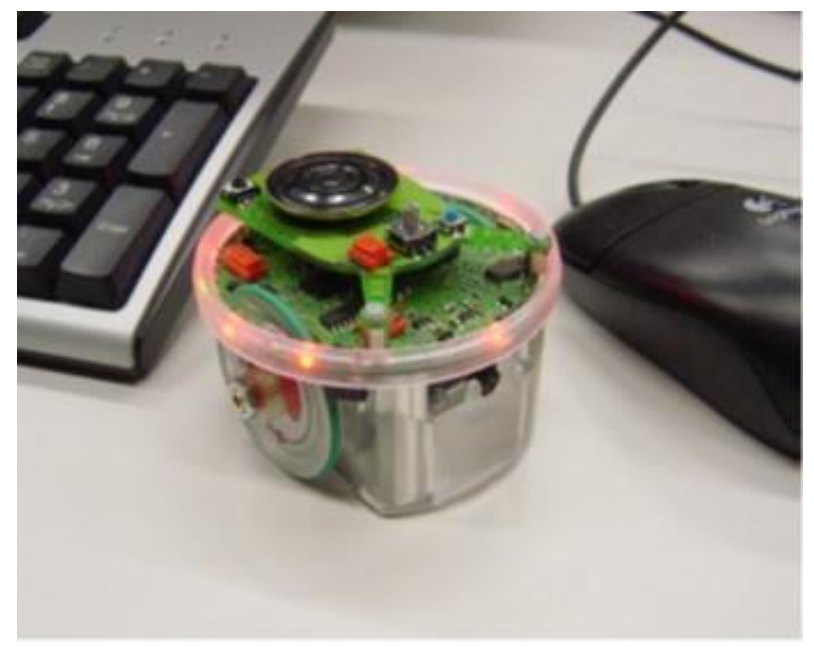

Şekil 7. E-Puck Robot (Cianci vd., 2009)

OpenBot (Müller \& Koltun, 2020), Müller ve Koltun tarafından Intel Laboratuvarlarında geliştirilmiş olup, geliştiriciler tarafından robot bilimi araştırmalarına ve eğitimine destek olmak amacıyla donanım ve yazılım dosyalarının açık kaynak olacağı bildirilmiştir. Herhangi bir üniversitenin mühendislik eğitiminde kullanımıyla ilgili bir bilgiye ulaşılamamıştır. Printbots (García-Saura \& GonzálezGómez, 2012), Garcia-Saura ve ark. tarafından eğitim amaçlı geliştirilen ve sunulan, açık donanım kaynağı olan gezgin 
robotlardır. Mayıs 2012 yılında, Autonoma de Madrid Üniversitesinde, Robotiğe Giriş dersinde kullanılmıştır. (Arvin vd., 2019)'da Vona ve ark. tüm donanımı ve yazılımı açık kaynaklı bir gezgin manipülatör robotu geliştirmişlerdir. Vona ile lisans ve yüksek lisans seviyesinde robot bilimi öğretilebilmesi için 5 farklı müfredat modülü hazırlanmıştır. (Betthauser vd., 2014)'te Betthauser ve ark. işlemci gücü açısından bilgisayarlı görü deneyleri için çok uygun olan, eğitim ve araştırma amaçlı, açık kaynaklı WolfBot‘u geliştirmişlerdir. (Karimi, Ahmadi, Korghond, Babaians, \& Ghidary, 2015)'te Karimi ve ark. eğitim ve araştırma amaçlı, dağıtık giriş-çıkış modüllerine sahip, donanım ve yazılım anlamında açık kaynaklı ReMoRo gezgin robotunu geliştirmişlerdir. Üniversitelerin mühendislik eğitiminde ReMoRo'nun kullanımıyla ilgili bir bilgiye rastlanmamıştır. (Guyonneau \& Mercier, 2019)‘da da Guyonneau ve Mercier, eğitim ve araştırma amaçlı geliştirdikleri donanım ve yazılımı tamamen açık kaynaklı IstiABot gezgin robotunu tanıtmışlardır. Bu robot, Angers Üniversitesinin mühendislik okulu olan Polytech Angers'daki gezgin robotlar eğitiminde kullanılmak üzere geliştirilmiştir. Veter robot ("Veter - robot for researchers", y.y.), Linorobot ("Linorobot", y.y.), NasaJPL Open Source Rover ("GitHub - nasa-jpl/open-sourcerover: A build-it-yourself, 6-wheel rover based on the rovers on Mars!”, y.y.), Arduino Robot (“Arduino Robot”, y.y.), Zumy ("Zumy — Biomimetic Millisystems Lab Collaboration Wiki”, у.у.), Leo Rover (“Leo Rover”, y.y.), LoCoBot ("LoCoBot · An Open Source Low Cost Robot", y.y.), ROSbot ("ROSbot", y.y.), Oculus Prime ("Oculus Prime", y.y.), Roch robot ("Roch robotSoy|Robotics|technology|SoyRobotics-Suzhou Soy Robot technology co. LTD”, y.y.) ve MAEBot (“APRIL Laboratory : Autonomy * Perception * Robotics * Interfaces * Learning", y.y.) da çeşitli üniversitelerde kullanılan veya kullanılabilecek şekilde üretilen açık kaynaklı gezgin robotlardır. Ek olarak ("OPENROBOTHARDWARE - An Initiative for Open Robot Hardware”, y.y.) de açık donanım kaynağı sunulan robotların paylaşıldığı bir web sitesidir. Gezgin robotların tekil kullanımlarından ziyade çoklu bir şekilde kullanımı üzerine de çalışmalar yapılmaktadır.

Pickem ve ark. (Pickem vd., 2017) sürü robot ve çoklu robot çalışmaları için tüm dünyanın uzaktan erişim ile kullanımına açık olan Robotarium projesini geliştirmişlerdir. 2017 yılında hizmete açılan çoklu robot deney setinde GritBot adı verilen donanım ve yazılım anlamında tamamen açık kaynaklı gezgin robotlar kullanılmaktadır. (Opportunities, Remote-access, Control, \& Multirobot, 2020)'deki çalışmanın yayınına kadar geçen süreçte tüm dünyadan yüzlerce kullanıcıya ve 1000 civarında deney sayısına ulaşan Robotarium Uygulaması pandemiden dolayı meydana gelen yoğunluk nedeniyle şu sıralar erişime kısıtlıdır.

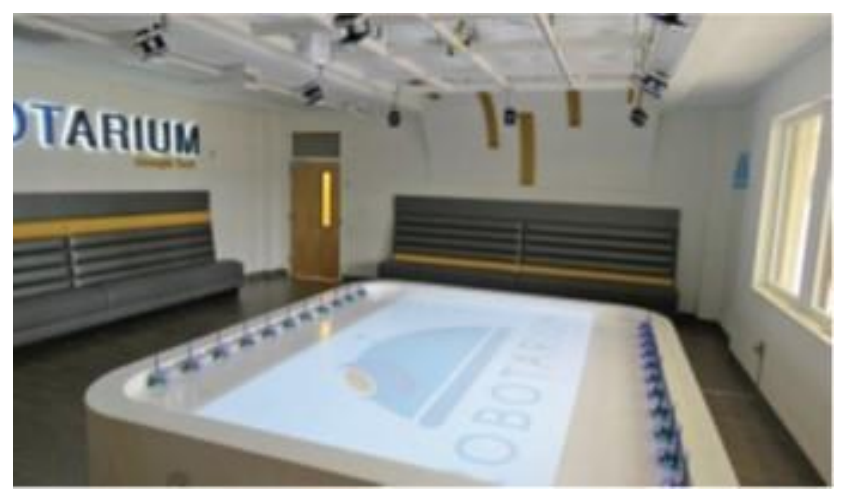

Şekil 8. GritBot X robotlarıyla dolu Robotarium Deney Seti (Opportunities vd., 2020)

Şekil 8‘de deney seti görülen Robotarium‘un iş akış döngüsü de şekil 9'da sunulmuştur.

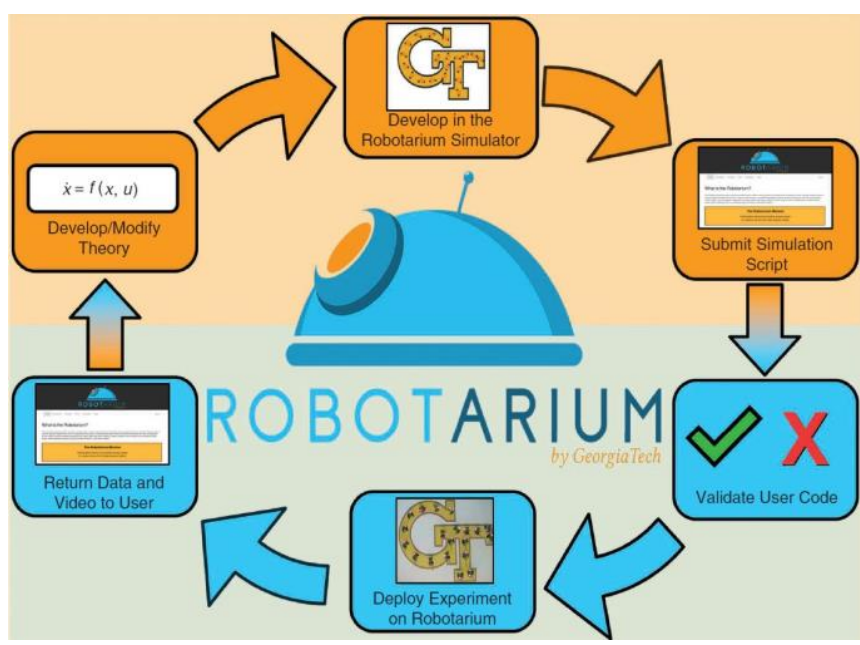

Şekil 9. Robotarium Proje Başvuru ve Sonuç Süreci (Opportunities vd., 2020)

$\mathrm{Bu}$ anlamda dünyanın ilk çoklu robot deney seti olan Robotarium'u, Duckietown projesi takip etmiştir. Duckietown'ın Robotarium'dan farkı gerçek bir şehir yapısı sunarak, daha çok bilgisayarlı görü ve yapay zekâ çalışmalarına olanak sağlamasıdır. Bir grup akademisyen tarafından MIT'de geliştirilen Duckietown projesi, yine MIT'de ETH Zürich ve Université de Montréal gibi çeşitli üniversitelerde yapay zekâ, bilgisayarlı görü algoritmalar gibi konuların öğretilmesi amacıyla derslerde kullanılmıştır ("Duckietown Foundation - Duckietown", y.y.). Gezgin robotlar denilince çokça akla gelen tekerlekli robotların dışında bacaklı robotlar da birer gezgin robottur. Tek bacaklı, iki bacaklı, dört ve daha çok bacaklı robotlar üzerine de çalışmalar yapılmaktadır. Ancak bu robotlar kaynaklarda genellikle bacaklı robotlar ya da insansı robotlar olarak geçmektedir. İnsansı robotları, gezgin robotlar alanının içinde, fakat farklı parametreleri de barındırdığından çok uzak bir köşesinde konumlandırdığım için bu çalışmaya dahil etmedim. Bacaklı hareket kabiliyetiyle ilgili araştırmalar için Grimminger ve ark. (Grimminger vd., 2020) tarafindan geliştirilen tork kontrollü modüler robot mimarisi, Oncilla (Grimminger vd., 2020) ve Stanford-doggo (Kau, Schultz, Ferrante, \& Slade, 2019) araştırmacılara önemli olanaklar sağlarken mühendislik eğitiminde kullanımına ilişkin henüz bir bilgiye ulaşılamamıştır. Köpek tipindeki bu robotların 
dışında yine dört bacaklı örümcek tipinde olan açık donanım kaynağı sunan Aracna (Lohmann vd., 2012) robot, Lohmann ve ark. tarafindan geliştirilmiştir. Diğer dört bacaklı robotlar gibi mühendislik eğitiminde kullanıldıklarına dair bir bilgiye ulaşılamamıştır.

\section{3. Örnek Bir Müfredat}

Gezgin robotların yaygınlaşmasıyla birlikte bu robotları üreten ve kullanan kişis sayısı da artmaya başlamıştır. Gelişmiş ve ticari amaçla üretilip satılan gezgin robotların üniversiteler veya daha kapsamlı bir şekilde ifade etmek gerekirse eğitim ve öğretim kurumları tarafından kolaylıkla alınıp kullanılamaması nedeniyle açık kaynaklı gezgin robotların üretilmesi ve yaygınlaştırılması gibi ihtiyaçlar doğmuştur. Çünkü gezgin robotların çeşitli sektörlerde aktif olarak kullanımıyla birlikte bu robotları kullanacak, bakımını ve tamirini yapacak uzman çalışan talebi ortaya çıkmıştır. Hem bu talebi karşılamak hem de gezgin robotlar alanını daha da geliştirecek nitelikli araştırmacıların yetişmesini sağlamak amacıyla üniversiteler, bazı eğitim ve öğretim kurumları gezgin robotlarla ilgili dersler vermek için müfredatlar hazırlamaktadır. $\mathrm{Bu}$ müfredatlar her geçen gün güncellenmekle birlikte aktif olarak üniversitelerin mühendislik eğitiminde uygulanmaktadır. $\mathrm{Bu}$ çalışmada, gezgin robotlarla ilgili veya gezgin robotların yoğunlukla kullanıldığı müfredatlar hazırlayıp, bunları mühendislik eğitiminde kullanan ulusal ve uluslararası önemli birçok üniversite incelenerek gezgin robotlar dersi için örnek bir müfredat hazırlanmıştır. İncelenen üniversitelerin listesi uluslararası ve ulusal olmak üzere iki alt başlık altında, 2021 Times Higher Education Dünya Üniversite Sıralamasındaki başarı sırasına göre aşağıda verilmiştir. Başarı sıraları üniversite adlarının sağında, parantez içinde belirtilmiştir.

\subsection{Uluslararası Üniversiteler}

1. Stanford Üniversitesi

2. Harvard Üniversitesi

3. California Teknoloji Enstitüsü

4. Massachusetts Teknoloji Enstitüsü

5. California Üniversitesi, Berkeley

6. Yale Üniversitesi

7. Princeton Üniversitesi

8. Johns Hopkins Üniversitesi

9. Pennsylvania Üniversitesi, Phila.

10. ETH Zürich Üniversitesi

11. California Üniversitesi, Los Angeles

12. Columbia Üniversitesi

13. Cornell Üniversitesi

14. Michigan Üniversitesi, Ann Arbor

15. Northwestern Üniversitesi

16. Carnegie Mellon Üniversitesi

17. Washington Üniversitesi, Seattle

18. Georgia Teknoloji Enstitüsü

19. EPFL

20. Texas Üniversitesi, Austin

21. KU Leuven

22. Washington Üniversitesi, St Louis

23. Manchester Üniversitesi

24. Southern California Üniversitesi

25. Brown Üniversitesi

26. Amsterdam Üniversitesi

27. Maryland Üniversitesi, College Park
28. Colorado Üniversitesi, Boulder

29. Sussex Üniversitesi

30. Massachusetts Üniversitesi, Amherst

31. Virginia Tech

32. Utah Üniversitesi

33. Southern Denmark Üniversitesi

34. Oregon Eyalet Üniversitesi

35. Colorado Maden Okulu

36. Worcester Polytechnic Enstitüsü

37. Vilnius Gediminas Teknik Üni.

38. Florida Tarım ve Mekanik Üni.

39. Southern Illinois Üniversitesi

40. Colorado Eyalet Üniversitesi

$(201-250)$

(201-250)

(251-300)

(401-500)

$(401-500)$

(601-800)

(801-1000)

(801-1000)

(801-1000)

$(-)$

3.2 Ulusal Üniversiteler

1. Koç Üniversitesi

2. Sabanc1 Üniversitesi

3. Hacettepe Üniversitesi

4. Boğaziçi Üniversitesi

5. Bilkent Üniversitesi

6. İstanbul Teknik Üniversitesi

7. Orta Doğu Teknik Üniversitesi

8. Karabük Üniversitesi

9. Marmara Üniversitesi

10. Yıldız Teknik Üniversitesi

11. Gazi Üniversitesi

12. Bahçeşehir Üniversitesi

13. Eskişehir Osmangazi Üniversitesi

14. Kocaeli Üniversitesi

15. İzmir Yüksek Teknoloji Üniversitesi

$(401-500)$

(501-600)

(501-600)

(601-800)

(601-800)

(801-1000)

(801-1000)

(801-1000)

$(1001+)$

$(1001+)$

$(1001+)$

$(1001+)$

$(1001+)$

$(-)$

16. Sakarya Uygulamalı Bilimler Üni.

17. Firat Üniversitesi

18. İstanbul Sabahattin Zaim Üniversitesi

19. Düzce Üniversitesi

Listedeki, mühendislik eğitiminde gezgin robotlarla ilintili dersler hakkında veya doğrudan gezgin robotlar dersleriyle ilgili daha fazla bilgi ve kaynak sunan ya da gezgin robotlara daha fazla yoğunlaşmış üniversitelerin ("racecar", y.y.), (Srinivasa vd., 2019), (Adinandra vd., 2018), (“COS 495: Lectures", y.y.), ("PENN Engineering - 2016-ROBOSyllabus", y.y.), (“Autonomous Mobile Robots | ETH Zürich”, y.y.), (“Official Page for NA 568/EECS 568/ROB 530 Mobile Robotics: Methods \& Algorithms”, у.у.), (Lalonde, Bartley, \& Nourbakhsh, 2006), (Croix \& Egerstedt, y.y.), ("Basics of mobile robotics | EPFL", y.y.), (Urtlebot \& Slaets, 2020), ("Mobile Robotics", у.у.), ("Education Robotics and Autonomous Systems Center", y.y.), ("Brown CS: CSCI1480", у.у.), ("Bachelor Artificial Intelligence Introduction to Autonomous Mobile Robots", y.y.), ("Master of Engineering Degree | Maryland Robotics Center", y.y.), ("FAMU-FSU EML 4830", y.y.), ("Autonomous Mobile Robotics", y.y.), ("Sabanc1 University :: Course Offerings", y.y.), ("CMPE 434 - Introduction to Robotics Course Home Page", y.y.), ("Bilkent University - Online Academic Catalog”, y.y.), (“ninova - İTÜ e-Öğrenim Merkezi”, y.y.-a), ("ninova - İTÜ e-Öğrenim Merkezi”, y.y.-b), ("CENG786 Robot Motion Planning and Control", y.y.), ("YTU - Bologna Information System - View Course", y.y.-a), ("YTU - Bologna Information System - View Course", y.y.-b), ("YTU Bologna Information System - View Course", y.y.-c), ("YTU - Bologna Information System - View Course", y.y.-d), 
("YTU - Bologna Information System - View Course", y.y.e), ("YTU - Bologna Information System - View Course", y.y.-f), ("BAU Robotics - Teaching | BAU | Bahçeşehir Üniversitesi”, y.y.), ("OGU - Int. To Autonomous Mobile Robots”, y.y.), (Parlaktuna, y.y.), ("Robotik Sistemlere Giriş Yapay Zekâ ve Benzetim Sistemleri Arge Lab", y.y.), ("Bilgisayar Bilimleri ve Mühendisliği (-30 İngilizce) | İZÜ", y.y.)'deki ders müfredatları, haftalık ders planları, tecrübeleri, yöntemleri vb. öğeleri incelenmiştir. Listedeki diğer üniversitelerde, mühendislik eğitiminde verilen gezgin robot ve ilintili derslerle ilgili yeterli bilgi ve kaynak sunulmamıştır. Ya gezgin robotlar genel robot bilimine giriş derslerinin bir veya iki haftalık konusu olarak çok kısa bir süreliğine ele alınmıştır ya gezgin robot kullanımı hiç olmamıştır, ya da gezgin robotlara yeteri kadar önem verilmemiştir. İncelenen ders müfredatlarının birçoğunda, gezgin robotlara yoğunlaşılan, robot bilimiyle ilgili derslerin alınabilmesi için Kalkülüs, Doğrusal Cebir vb. matematiksel derslerin, Algoritma ve Veri Yapıları, Programlama gibi derslerin alınması üniversiteler tarafindan ön koşul olarak istenmekle birlikte, Python, $\mathrm{C}++$ gibi programlama dillerinin bir veya ikisinin bilinmesi ve GNU/Linux işletim sistemiyle rahatlıkla çalış1labilmesi de yine üniversiteler tarafından önemle tavsiye edilmektedir. Örneğin ("Official Page for NA 568/EECS 568/ROB 530 Mobile Robotics: Methods \& Algorithms", y.y.) ve ("CSE 478: Autonomous Robotics", y.y.)'e bakılabilir. Genel olarak müfredatlarla ilgili şöyle bir sonuca varılmıştır: Müfredatların ilk haftalarında giriş dersleri, matematiksel arka plan, gezgin robot kinematiği, dinamiği, sürüş tipleri gibi konular işlenmektedir. Akabinde algılayıcılar, elektronik parçalar, ile ilgili bilgiler verilmektedir. Algılayıcıların ardından ders boyunca kullanılacak yazılım araçları (ROS, Matlab vb.) ve benzetim ortamları (Gazebo, Webot vb.) tanıtılmaktadır. Gezgin robotlarda doğrusal hareket, dönme hareketi, hedefe gitme, yön bulma gibi çeşitli kontrol algoritmaları devam eden haftalarda anlatılmaktadır. Gezgin robotlarla çalışma deneyiminin kazanılmasının ardından daha ileri konulara giriş yapılmaktadır. Bunlar: Konumlandırma, Yön Bulma Yı̆̆ını (Rota Planlama ve Engelden Kaçınma), Haritalama ve İngilizce kaynaklarda "SLAM (Simultaneous Localization And Mapping)" olarak bilinen Eş Zamanlı Konumlandırma ve Haritalama algoritmalarıdır. Bunu son haftalarda sürü robotlar, çoklu robotlar ya da yapay zekâ çalışmaları ve denemeleri izlemektedir. Dersler genellikle uygulama ağırlıklı geçmekte olup, öğrenciler belirli takımlara bölünmektedir. Belirli aralıklarla bu takımlardan küçük projeler, sunumlar istenmektedir. Öğrenciler bu projeleri yaparak işin içine daha çok girmektedirler. Son olarak da öğrencilerden robot yarışmalarına katılabilecekleri bir final projesi yapmaları istenmektedir. Bu da öğrencileri motive eden güzel bir araçtır. Bu çalışmada, incelenen müfredatlar ışı̆̆ında gezgin robotlar dersi için örnek bir müfredat hazırlanmıştır. Yapılan araştırmalar sonucu bazı üniversitelerin akademik dönemlerinin diğerlerinden farklı olduğu gözlemlenmiştir. Ancak büyük bölümünün bir akademik dönemi 15 haftadır. Dolayısıyla son haftası proje sunumu olacak şekilde 15 haftalık örnek bir müfredat oluşturulmuştur. Oluşturulan müfredat haftalık sırasıyla aşağıdaki gibidir:

1. Hafta: Gezgin Robotlara Giriş

2. Hafta: Gezgin Robot Kinematiği ve Matematiksel
Arka Plan

3. Hafta: Yazılım Araçları ve Benzetim Ortamına Giriş (ROS ‘a ve Gazebo‘ya Giriş)

4. Hafta: Turtlesim Kullanımı ve Gazebo Benzetim Ortamına Robot yükleme, RQT (RQT: ROS visualization tool based on Quasar Technologies) Grafiği ve Kullanımı

5. Hafta: Alıcılar (Sensörler)'a ve Algılama (Perception)'ya Giriş, Temel ve Gelişmiş Alıcı (Sensör) Türleri ve Çalışma İlkeleri

6. Hafta: Bilgisayarlı Görü‘ye Giriş

7. Hafta: Ara Sinav

8. Hafta: Hareket ve Kontrole Giriş

9. Hafta: Doğrusal, Dönel ve Hibrit Hareket, Odyometre Bilgisi

10. Hafta: P, PI, PD ve PID Kontrol

11. Hafta: Konumlandırma, Planlama, Yön Bulma ve Haritalama Algoritmalarına Giriş

12. Hafta: Konumlandırma ve Yön Bulma

13. Hafta: Haritalama ve SLAM Algoritmaları

14. Hafta: Final Sinavi

15. Hafta: Final Proje Sunumları ya da Yarışma Haftası

Gezgin Robotlar dersi bir dönem boyunca, haftalık 3 saat olacak şekilde düşünülmüştür. Bu 3 saatlik dersin ilk 3 hafta boyunca teorik olarak, sonraki haftalarda ise 1 saati teorik ve kalan 2 saati ise uygulama olarak düşünülmüştür. Dersin işleneceği dönem boyunca öğrencilerin toplamda 10 adet deney, uygulama ve 1 adet de final projesi yapmasi düşünülmüştür. Oluşturulan haftalık planı detaylandırmak gerekirse, ilk haftada Gezgin Robotların kısa tarihçesi, gelişim süreçleri, güncel durumu, kullanım alanlarıyla ilgili bilgiler verilmektedir. Bunun yanı sıra öğrencilerden üçerli takımlar oluşturmaları istenmektedir. (Lalonde vd., 2006)'da Lalonde ve ark. 3'ten fazla öğrenciyle oluşturulan takımların yararlı olmadığını ve takım içinde gruplaşmalar olduğunu belirtmişlerdir. O yüzden üçerli takımlar idealdir. Ek olarak, öğrencilere ders izlencesi, final projesi ve değerlendirme (not verme) kriterleri açıklanmaktadır. İkinci hafta gezgin robot kinematiğine giriş yapılmaktadır. Tek Teker Sürüş, Diferansiyel Sürüş, Ackermann Sürüş ve Omni Sürüş ile ilgili bilgiler verilip kinematik analizleri yapılmaktadır. Yine dönem boyunca gerekli olan matematiksel arka plan da bu derste verilir. Ardından öğrencilerin yavaş yavaş dersin içine girmelerini sağlamak amacıyla yazılım araçlarından bahsedilir. Benzetim ortamları tanıtılır. Bu derste kullanılacak olan ROS ve Gazebo araçlarının kurulumu yapılır. Sanal çalışma ortamı oluşturulur. 4. haftaya gelindiğinde artık teorik ders 1 saat, uygulama ise 2 saate çıkar. Burada amaç öğrencinin dinleyerek değil yaparak öğrenmesini sağlamaktır. Turtlesim Kullanımı ve Gazebo'ya robot yükleme, RQT Grafikleri gibi konulardan bahsedilir. Ardından öğrencilerden önce Turtlesimde basit bir gezgin robot uygulaması yapmaları, (Ör. Kare, daire şeklinde bir gezinti yapması) daha sonra da aynı uygulamayı Gazebo 'da yapmaları istenir. Burada öğrenciler, .world, .launch uzantılı dosyalarla ilgili bilgiler öğrenirken ilk uygulamalarını da yapmış olurlar. 5. haftayla birlikte alıcılara ve algılamaya giriş yapılır. Temel ve gelişmiş alıcı türleri ve çalışma ilkelerinden bahsedilir. Ultrasonik, kızılötesi gibi temel alıcıların yanı sıra IMU (Inertial Measurement Unit), GPS (Global Positioning System), LIDAR (Light Detection and Ranging), RGBD (Red Green 
Blue Depth) Kamera gibi gelişmiş alıcıların çalışma ilkeleri anlatılır. Dersin uygulama kısmında, edinmesi daha az maliyetli olduğundan temel alıcılarla ilgili uygulamalar yapılır. Sonraki hafta Bilgisayarlı Görü'ye giriş yapılarak kamerayla algılama yapılması ile ilgili uygulamalar yapılır. 7. haftayla birlikte ara sinavlar yapılır. Ara sinavlardan sonra hareket ve kontrol konularına giriş yapılır. Yine gerekli temel bilgiler verilerek daha önce Turtlesimde yapılan örnek gezinti uygulamaları gerçek gezgin robotlarla yapılır. 9. haftada doğrusal, dönel ve hibrit hareketlerin yanında odyometre bilgisiyle ilgili de çalışmalar ve uygulamalar yapılır. Sonraki haftayla birlikte daha olgun bir robot çalışması yapabilmek adına temel kontrol yapıları anlatılır. Öğrencilerden kontrolcü tasarımları yapmaları ve tasarladıkları kontrolcüleri gerçek robotta uygulamaları istenir. 11. haftada ise gezgin robotlarda daha ileri konulara giriş yapılır. Bunlar; konumlandırma, planlama, yön bulma, haritalama gibi konulardır. Burada, kullanılan algoritmalardan bahsedilir. $\mathrm{Bu}$ algoritmaların benzetim ortamındaki robotlarda uygulanması istenir. Sonraki haftalarda da daha detaylı olarak bu algoritmalar incelenir ve gerçek robotlarda uygulamaları yapılır. Son olarak da EZKH (Eş Zamanlı Konumlandırma ve Haritalama) algoritmaları denenir. Final sınavlarının ardından öğrencilerin yaptıkları final projeleri sunulur. (Avanzato, 2002)`deki gibi bazı üniversiteler final projesi olarak öğrencilerin bir gezgin robot yarışmasına katılacak robotu yapmalarını istemektedir. Bu da bir tercihtir. Haftalık ders planı ve uygulamaların yanı sıra (Piepmeier, Bishop, \& Knowles, 2003)'te Piepmeier ve ark.ının da bahsettiği gibi her hafta bir öğrenci takımından o hafta, robot bilimi ve gezgin robotlar alanında yaşanan gelişmelerle ilgili medyaya yansıyan bilgilerden oluşan bir haber kuşağı yapmaları ve bunu dersin ilk 5 dakikasında tüm sınıfa sunmaları istenmektedir. Piepmeier ve ark. buna "Robot Haberleri (Robot News)" demektedirler. Bu, öğrencilerin medya platformlarını kendi alanları için araştırmalarını sağlayarak onlara robot bilimi alanındaki yeni gelişmeleri takip etme imkânı verecektir. Bunun yanı sıra yine (Piepmeier vd., 2003) Piepmeier ve ark. gibi her hafta robot bilimiyle ilgili bir film tavsiyesinin ve buna ilaveten kitap okumayı seven öğrenciler için de kitap tavsiyesi yapılmasının faydalı olacağı düşünülmektedir. Ayrıca tüm bunların dışında, mühendislik eğitimi veren öğretim görevlileri, yetiştirdikleri mühendislere etik ve insani değerleri de aşılamalı ve öğretmelidir. Bunun için (Hung \& Member, 1998)'da "The Ethics Challange" masa oyununun oynanması tavsiye edilmiştir. Bu oyunla öğrencilerin, etik değerleri eğlenerek öğrenmesi amaçlanmaktadır. Ayrıca dönem başında oluşturulan takımlar da öğrencilerin iletişim, takım çalışması gibi yumuşak becerilerinin gelişmesini sağlayacaktır.

\section{Sonuç}

Bu çalışmada 2021 Times Higher Education Dünya Üniversite Sıralamasında ilk 1000 'de ve dışında bulunan, mühendislik eğimi veren, ulusal ve uluslararası birçok üniversitenin robot bilimiyle ve gezgin robotlarla ilgili dersleri ve bu derslerin müfredatları incelenmiştir. $\mathrm{Bu}$ incelemeler açık kaynaklı gezgin robotların mühendislik eğitiminde kullanılması ve örnek bir müfredat oluşturulması bağlamında yapılmıştır. Bu süreçte $40^{`} 1$ uluslararası ve $19^{`} \mathrm{u}$ ulusal olmak üzere toplam 59 üniversite incelenmiştir. $\mathrm{Bu}$ üniversitelerin, eğer varsa mühendislik eğitiminde kullandıkları açık kaynaklı gezgin robotlar belirlenmiştir. Bunların dışında, üniversitelerin ilgili müfredatları incelenmiş ve örnek bir gezgin robotlar müfredatı hazırlanmıştır. Üniversitelerin büyük çoğunluğu dersleri, uygulama ve proje ağırlıklı hazırlarken bazıları da sadece giriş seviyesinde teorik ağırlıklı dersler halinde bırakmaktadır. Robot bilimine ve gezgin robotlara ağırlık veren üniversiteler her yıl, bir önceki yıldan elde ettikleri deneyime ve yeni bilimsel yöntemlere göre müfredatlarını güncellemektedir. Ancak, gelişmelerin artış hızı bu şekilde devam ederse çoğunlukla bir dönemde verilen gezgin robotlar dersi, ilerleyen yıllarda iki dönemde verilebilir.

\section{Kaynaklar}

About - TurtleBot. (y.y.). Tarihinde 21 Ocak 2021, adresinden erişildi https://www.turtlebot.com/about/

About the GNU Project - GNU Project - Free Software Foundation. (y.y.). Tarihinde 20 Ocak 2021, adresinden erişildi https://www.gnu.org/gnu/thegnuproject.en.html\#top

Adinandra, S., Nofriyudi, F., Pratama, A. W., \& Ratnawati, D. A. (2018). CEPI: A low cost and open source mobile robot platform for research and education. AIP Conference Proceedings, 1977(June). https://doi.org/10.1063/1.5042913

Albo-Canals, J., Garcia-Casulleras, M., De Cordoba, D., Canaleta, X., \& Gonzalez-Dachs, E. (2014). The educational robotic platform LSMaker EV1: Standardization vs customization. Iberian Conference on Information Systems and Technologies, CISTI, 1-5. https://doi.org/10.1109/CISTI.2014.6877005

APRIL Laboratory : Autonomy * Perception * Robotics * Interfaces * Learning. (y.y.). Tarihinde 21 Ocak 2021, adresinden erişildi https://april.eecs.umich.edu/maebot/

Arduino Robot. (y.y.). Tarihinde 21 Ocak 2021, adresinden erişildi https://store.arduino.cc/usa/arduino-robot

Arvin, F., Espinosa, J., Bird, B., West, A., Watson, S., \& Lennox, B. (2019). Mona: an Affordable Open-Source Mobile Robot for Education and Research. Journal of Intelligent and Robotic Systems: Theory and Applications, 94(3-4), 761775. https://doi.org/10.1007/s10846-018-0866-9

Autonomous Mobile Robotics. (y.y.). Tarihinde 21 Ocak 2021, adresinden erişildi https://www2.cs.siu.edu/ hexmoor/classes/CS404S17/robot17.html

Autonomous Mobile Robots | ETH Zürich. (y.y.). Tarihinde 21 Ocak 2021, adresinden erişildi https://online.ethz.ch/courses/coursev1:ETH+AMRx_FS2020+2020_T1/about

Avanzato, R. (2002). Mobile robotics for freshman design, research, and high school outreach. 736-738. https://doi.org/10.1109/icsmc.2000.885083

Bachelor Artificial Intelligence - Introduction to Autonomous Mobile Robots. (y.y.). Tarihinde 21 Ocak 2021, adresinden erişildi https://staff.fnwi.uva.nl/a.visser/education/AutonomousMobi leRobots/

Basics of mobile robotics | EPFL. (y.y.). Tarihinde 21 Ocak 2021, adresinden erişildi https://edu.epfl.ch/coursebook/en/basicsof-mobile-robotics-MICRO-452 
BAU Robotics - Teaching | BAU | Bahçeşehir Üniversitesi. (y.y.). Tarihinde 21 Ocak 2021, adresinden erişildi https://bau.edu.tr/icerik/11681-teaching

Betthauser, J., Benavides, D., Schornick, J., O’Hara, N., Patel, J., Cole, J., \& Lobaton, E. (2014). WolfBot: A distributed mobile sensing platform for research and education. Proceedings of the 2014 Zone 1 Conference of the American Society for Engineering Education - "Engineering Education: Industry Involvement and Interdisciplinary Trends", ASEE Zone 1 2014, 1-8. https://doi.org/10.1109/ASEEZone1.2014.6820632

Bilgisayar Bilimleri ve Mühendisliği (-30 İngilizce) | İZÜ. (у.y.). Tarihinde 21 Ocak 2021, adresinden erişildi https://www.izu.edu.tr/akademik/enstituler/lisansustuegitim-enstitusu/programlar/doktora/bilgisayar-bilimleri-vemuhendisligi-30-ingilizce

Bilkent University - Online Academic Catalog. (у.y.). Tarihinde 21 Ocak 2021, adresinden erişildi https://catalog.bilkent.edu.tr/course/c16574.html

Brown CS: CSCI1480. (y.y.). Tarihinde 21 Ocak 2021, adresinden erişildi https://cs.brown.edu/courses/info/csci1480/

CENG786 Robot Motion Planning and Control. (y.y.). Tarihinde 21 Ocak 2021, adresinden erişildi http://user.ceng.metu.edu.tr/ saranli/courses/ceng786/

Cianci, C., Magnenat, S., Pugh, J., Alves, C. M. O., Raemy, X., Zufferey, J.-C., ... Bonani, M. (2009). The e-puck, a Robot Designed for Education in Engineering. Proceedings of the 9th Conference on Autonomous Robot Systems and Competitions, 1(1), 59-65. Tarihinde adresinden erişildi http://infoscience.epfl.ch/record/135236

CMPE 434 - Introduction to Robotics Course Home Page. (y.y.). Tarihinde 21 Ocak 2021, adresinden erişildi http://robot.cmpe.boun.edu.tr/ cmpe434/doku.php?id=home

COS 495: Lectures. (y.y.). Tarihinde 21 Ocak 2021, adresinden erişildi https://www.cs.princeton.edu/courses/archive/fall11/cos495/ lectures.html

Croix, J. De, \& Egerstedt, M. (y.y.). Flipping the Controls Classroom Around a MOOC.

CSE 478: Autonomous Robotics. (y.y.). Tarihinde 21 Ocak 2021, adresinden erişildi https://courses.cs.washington.edu/courses/cse478/20wi/

Duckietown Foundation - Duckietown. (y.y.). Tarihinde 21 Ocak 2021, adresinden erişildi

https://www.duckietown.org/about/duckietown-foundation

Education - Robotics and Autonomous Systems Center. (y.y.). Tarihinde 21 Ocak 2021, adresinden erişildi https://rasc.usc.edu/education/

Evarobot. (у.у.). Tarihinde 21 Ocak 2021, adresinden erişildi http://www.evarobot.info/

FAMU-FSU EML 4830. (y.y.). Tarihinde 21 Ocak 2021, adresinden erişildi https://eng.famu.fsu.edu/sites/default/files/pdfs/me/CourseS yllabi/2015/EML4830.pdf

García-Saura, C., \& González-Gómez, J. (2012). Low cost educational platform for robotics, using open-source $3 \mathrm{~d}$ printers and open-source hardware. ICERI2012 Proceedings, (November), 2699-2706. Tarihinde adresinden erişildi https://library.iated.org/view/GARCIASAURA2012LOW
GitHub - nasa-jpl/open-source-rover: A build-it-yourself, 6-wheel rover based on the rovers on Mars! (y.y.). Tarihinde 21 Ocak 2021, adresinden erişildi https://github.com/nasa-jpl/opensource-rover

Grimminger, F., Meduri, A., Khadiv, M., Viereck, J., Wuthrich, M., Naveau, M., ... Righetti, L. (2020). An Open TorqueControlled Modular Robot Architecture for Legged Locomotion Research. IEEE Robotics and Automation Letters, 5(2), 3650-3657. https://doi.org/10.1109/LRA.2020.2976639

Guyonneau, R., \& Mercier, F. (2019). IstiABot, an open source mobile robot for education and research. 12th International Workshop on Robot Motion and Control, RoMoCo 2019 Workshop Proceedings, 131-136. https://doi.org/10.1109/RoMoCo.2019.8787363

How I coined the term "open source" | Opensource.com. (y.y.). Tarihinde 20 Ocak 2021, adresinden erişildi https://opensource.com/article/18/2/coining-term-opensource-software

Hung, J. Y., \& Member, S. (1998). An Integrated Junior-Year Laboratory Based on an Autonomous Mobile Robot Platform. 1154-1159.

Karimi, M., Ahmadi, A., Korghond, N. K., Babaians, E., \& Ghidary, S. S. (2015). ReMoRo; A mobile robot platform based on distributed I/O modules for research and education. International Conference on Robotics and Mechatronics, ICROM 2015, (Id), 657-662. https://doi.org/10.1109/ICRoM.2015.7367861

Kau, N., Schultz, A., Ferrante, N., \& Slade, P. (2019). Stanford Doggo: An open-source, quasi-direct-drive quadruped. arXiv.

Lalonde, J. F., Bartley, C. P., \& Nourbakhsh, I. (2006). Mobile robot programming in education. Proceedings - IEEE International Conference on Robotics and Automation, 2006(May), 345-350. https://doi.org/10.1109/ROBOT.2006.1641735

Leo Rover. (y.y.). Tarihinde 21 Ocak 2021, adresinden erişildi https://robots.ros.org/leo-rover/

Linorobot. (у.y.). Tarihinde 21 Ocak 2021, adresinden erişildi https://linorobot.org/

LoCoBot · An Open Source Low Cost Robot. (y.y.). Tarihinde 21 Ocak 2021, adresinden erişildi http://www.locobot.org/

Lohmann, S., Yosinski, J., Gold, E., Clune, J., Blum, J., \& Lipson, H. (2012). Aracna: An open-source quadruped platform for evolutionary robotics. Artificial Life 13: Proceedings of the 13th International Conference on the Simulation and Synthesis of Living Systems, ALIFE 2012, (July 2012), 387-392. https://doi.org/10.7551/978-0-262-31050-5-ch051

Master of Engineering Degree | Maryland Robotics Center. (y.y.). Tarihinde 21 Ocak 2021, adresinden erişildi https://robotics.umd.edu/education/master-engineeringdegree

Mehmet Akçakoca. (2017). Eğitim ve Araştırma Amaçlı Gezgin Robot Geliştirilmesi.

mit-racecar · GitHub. (y.y.). Tarihinde 21 Ocak 2021, adresinden erișildi https://github.com/mit-racecar

Mobile Robotics. (y.y.). Tarihinde 21 Ocak 2021, adresinden erişildi https://classes.engineering.wustl.edu/cse550/index.php 
Müller, M., \& Koltun, V. (2020). OpenBot: Turning smartphones into robots. arXiv.

ninova - İTÜ e-Öğrenim Merkezi. (y.y.-a). Tarihinde 21 Ocak 2021, adresinden erişildi https://ninova.itu.edu.tr/tr/dersler/bilgisayar-bilisimfakultesi/4712/blg-456e/form

ninova - İTÜ e-Öğrenim Merkezi. (y.y.-b). Tarihinde 21 Ocak 2021, adresinden erişildi https://ninova.itu.edu.tr/tr/dersler/fen-bilimlerienstitusu/6862/kom-613e/form

Oculus Prime. (y.y.). Tarihinde 21 Ocak 2021, adresinden erişildi https://robots.ros.org/oculus-prime/

Official Page for NA 568/EECS 568/ROB 530 Mobile Robotics: Methods \& Algorithms. (y.y.). Tarihinde 21 Ocak 2021, adresinden erişildi http://robots.engin.umich.edu/mobilerobotics/

OGU - Int. To Autonomous Mobile Robots. (y.y.). Tarihinde 21 Ocak 2021, adresinden erişildi https://web.ogu.edu.tr/storage/ceng/Uploads/152117106IntT oAutonomousMobileRobots.pdf

Open Source - TurtleBot. (y.y.). Tarihinde 21 Ocak 2021, adresinden erişildi https://www.turtlebot.com/opensource/

OPENROBOTHARDWARE - An Initiative for Open Robot Hardware. (y.y.). Tarihinde 21 Ocak 2021, adresinden erişildi http://www.openrobothardware.org/

Opportunities, G. I., Remote-access, L. L. I. N., Control, D., \& Multirobot, O. F. (2020). The Robotarium. (February).

Parlaktuna, O. (y.y.). Eskişehir Osmangazi Üniversitesinde Gezgin Robotlara Yönelik Lisansüstü Eğitim Programı ElektrikElektronik Mühendisliği Bölümü.

PENN Engineering - 2016-ROBO-Syllabus. (y.y.). Tarihinde 21 Ocak 2021, adresinden erișildi https://esap.seas.upenn.edu/wpcontent/uploads/2015/06/2016-ROBO-Syllabus.pdf

Pickem, D., Glotfelter, P., Wang, L., Mote, M., Ames, A., Feron, E., \& Egerstedt, M. (2017). The Robotarium: A remotely accessible swarm robotics research testbed. Proceedings IEEE International Conference on Robotics and Automation, 1699-1706. https://doi.org/10.1109/ICRA.2017.7989200

Piepmeier, J. A., Bishop, B. E., \& Knowles, K. A. (2003). Modern robotics engineering instruction. IEEE Robotics and Automation Magazine, 10(2), 33-37. https://doi.org/10.1109/MRA.2003.1213614

Platform. (у.у.). Tarihinde 21 Ocak 2021, adresinden erişildi https://racecar.mit.edu/platform

racecar. (y.y.). Tarihinde 21 Ocak 2021, adresinden erişildi https://racecar.mit.edu/

Robotik Sistemlere Giriş - Yapay Zeka ve Benzetim Sistemleri Arge Lab. (y.y.). Tarihinde 21 Ocak 2021, adresinden erişildi http://yapbenzet.kocaeli.edu.tr/robotik-sistemleregiris-2/

Roch robot-Soy|Robotics|technology|SoyRobotics-Suzhou Soy Robot technology co. LTD. (y.y.). Tarihinde 21 Ocak 2021, adresinden erişildi http://www.soyrobotics.com/product/showproduct.php?lang $=$ en\&id $=107$

ROSbot. (y.y.). Tarihinde 21 Ocak 2021, adresinden erișildi https://robots.ros.org/rosbot/
Sabanc1 University :: Course Offerings. (y.y.). Tarihinde 21 Ocak 2021, adresinden erişildi https://www.sabanciuniv.edu/syllabus/courses.cfm?year=20 $20 \&$ term $=01 \&$ subject $=$ ME \& code $=425 \&$ lan $=$ eng

Srinivasa, S. S., Lancaster, P., Michalove, J., Schmittle, M., Summers, C., Rockett, M., ... Sadeghi, F. (2019). MuSHR: A low-cost, open-source robotic racecar for education and research. arXiv.

TurtleBot. (у.у.). Tarihinde 21 Ocak 2021, adresinden erişildi https://www.turtlebot.com/

Urtlebot, T., \& Slaets, P. (2020). Turtlebot 3 as a Robotics Education Platform. (October 2019). https://doi.org/10.1007/978-3-030-26945-6

Veter - robot for researchers. (у.у.). Tarihinde 21 Ocak 2021, adresinden erişildi https://veterobot.com/

YTU - Bologna Information System - View Course. (y.y.-a). Tarihinde 21 Ocak 2021, adresinden erişildi http://www.bologna.yildiz.edu.tr/index.php?r=course/view\& id $=9375 \&$ aid $=5$

YTU - Bologna Information System - View Course. (y.y.-b). Tarihinde 21 Ocak 2021, adresinden erişildi http://www.bologna.yildiz.edu.tr/index.php?r=course/view\& id $=5069 \&$ aid $=33$

YTU - Bologna Information System - View Course. (y.y.-c). Tarihinde 21 Ocak 2021, adresinden erişildi http://www.bologna.yildiz.edu.tr/index.php?r=course/view\& id $=6244 \&$ aid $=3$

YTU - Bologna Information System - View Course. (y.y.-d). Tarihinde 21 Ocak 2021, adresinden erișildi http://www.bologna.yildiz.edu.tr/index.php?r=course/view\& id $=1659 \&$ aid $=18$

YTU - Bologna Information System - View Course. (y.y.-e). Tarihinde 21 Ocak 2021, adresinden erişildi http://www.bologna.yildiz.edu.tr/index.php?r=course/view\& $\mathrm{id}=9429 \&$ aid $=3$

YTU - Bologna Information System - View Course. (y.y.-f). Tarihinde 21 Ocak 2021, adresinden erişildi http://www.bologna.yildiz.edu.tr/index.php?r=course/view\& id $=6900 \&$ aid $=3$

Zumy - Biomimetic Millisystems Lab Collaboration Wiki. (y.y.). Tarihinde 21 Ocak 2021, adresinden erişildi https://wiki.eecs.berkeley.edu/biomimetics/Main/Zumy 